Moreno, N., Alvarado, M., Angulo, R. G. \& Briceño, E. C. (2020). Una aproximación al estudio del teorema de Pitágoras con estudiantes de secundaria. Revista Latinoamericana de Etnomatemática, 13(2), 5-24. DOI: $10.22267 /$ relatem.20132.58

Artículo recibido el 13 de agosto de 2019. Aceptado para publicación el 31 de julio de 2020

\title{
Una aproximación al estudio del teorema de Pitágoras con estudiantes de secundaria
}

\section{An approach to the study of the Pythagorean theorem with high school students}

\author{
Nehemías Moreno Martínez $z^{1}$ \\ Marcelino Alvarado García ${ }^{2}$ \\ Rita Guadalupe Angulo Villanueva ${ }^{3}$ \\ Eduardo Carlos Briceño Solís ${ }^{4}$
}

\begin{abstract}
Resumen
Se describe una investigación cualitativa de caso, con un grupo de quince estudiantes de secundaria del estado de San Luis Potosí, México, acerca de una experiencia en aula donde se abordó una aproximación al estudio del teorema de Pitágoras, en la que se analizó la relación entre los lados de un triángulo rectángulo. Apoyados en la Socioepistemología, se diseñaron situaciones variacionales al adaptar al aula la elaboración y uso de las tolvas que se construyen en la industria de la Pailería. Las estrategias variacionales de comparación, seriación, predicción y estimación realizadas por los alumnos, sobre los datos que obtuvieron al medir los lados de un triángulo rectángulo visualizado al interior de las tolvas, les permitieron resignificar, en términos del almacenamiento y vaciado de material granulado de las tolvas, la relación matemática señalada por el teorema.
\end{abstract}

Palabras clave: Teorema de pitágoras; estrategia variacional; socioepistemología; educación secundaria.

\begin{abstract}
A qualitative case study is described, with a group of fifteen high school students from the state of San Luis Potosí, Mexico, about a classroom experience where an approach to the study of the Pythagorean theorem was addressed, in which the relationship between the sides of a right triangle was analyzed. Supported in Socioepistemology, variational situations were designed by adapting to the classroom the elaboration and use of the hoppers that are built in the Pailería industry. The variational strategies of comparison, seriation, prediction and estimation carried out by the students, on the data obtained when measuring the sides of a right triangle visualized inside the hoppers, allowed them to resignify, in terms of the storage and emptying of granulated material of the hoppers, the mathematical relation indicated by the theorem.

1 Dr. en Matemática Educativa. Universidad Autónoma de San Luis Potosí, San Luis Potosí, México. nehemias_moreno@live.com.

${ }^{2}$ Lic. en Matemática Educativa. Universidad Autónoma de San Luis Potosí, San Luis Potosí, México. marce 341@hotmail.com .

3 Dra. en Pedagogía. Universidad Autónoma de San Luis Potosí, San Luis Potosí, México. rita.angulo@uaslp.mx .

${ }^{4}$ Dr. en matemática educativa. Universidad Autónoma de Zacatecas, Zacatecas. ecbs74@gmail.com .
\end{abstract}


Key words: Pythagoras theorem; variational strategy; socioepistemology; secondary education.

\section{INTRODUCCIÓN}

Esta investigación aborda el problema de la enseñanza del Teorema de Pitágoras (TP), centrada en su definición y aplicación de la fórmula $a^{2}+b^{2}=c^{2}$. Consideramos que, antes de abordar el TP, es necesario que el estudiante advierta una relación entre los lados del triángulo rectángulo señalada en el TP mediante el planteamiento de situaciones que se apoyan en aspectos sociales, históricos, culturales e institucionales que también se encuentran involucrados en la generación del conocimiento matemático (Cantoral, ReyesGasperini \& Montiel, 2014).

En este trabajo se investigó una experiencia en aula con estudiantes de secundaria donde se abordó una aproximación al estudio del TP, para resignificar la relación entre los lados de un triángulo rectángulo, apoyándonos en el empleo y adaptación del proceso de construcción de tolvas, que se realiza en la industria de la Pailería, y del uso cotidiano de éstas para el almacenamiento y vaciado de granos por gravedad. De este modo, la pregunta que orientó la investigación fue ¿Cómo resignifican los estudiantes la relación entre los lados de un triángulo rectángulo señalada por el TP en el contexto de la construcción y uso de las tolvas?

\section{LA MATEMÁTICA QUE "VIVE" EN LA INDUSTRIA DE LA PAILERÍA}

La Pailería es un oficio que responde a la demanda de diferentes sectores industriales a través de la fabricación de estructuras metálicas formadas mediante el ensamble de piezas metálicas (láminas o placas que tienen al hierro como componente principal) sobre las que se han practicado diferentes cortes o dobleces. Algunos productos que se elaboran en Pailería son puentes, tolvas, tuberías, naves industriales, entre otras.

En el inicio de la investigación, uno de los autores del presente trabajo realizó una estancia de un mes en una empresa de Pailería en San Luis Potosí, México, donde llevó a cabo un método de trabajo de tipo etnomatemático donde observó las prácticas que realizaban algunos paileros enfocándose en qué hacen y cómo lo hacen, lo cual permitió elaborar una narrativa acerca de los elementos matemáticos presentes en sus prácticas (D’Ambrosio, 2014). Los paileros miden, trazan, cortan y ensamblan de inicio a fin los productos. La mayoría de los 
paileros no cuenta con estudios de primaria, sin embargo, esto no les impide construir estructuras metálicas donde se pone en juego el pensamiento matemático.

\subsection{La construcción de Tolvas}

En la estancia realizada en la empresa se observó el proceso de construcción de algunas tolvas, figura 1(a) y (b). La forma geométrica y el uso (que depende de su forma) de la tolva es variada. En particular, se observó el proceso de construcción de la tolva con forma de pirámide truncada, la cual es empleada para almacenar y vaciar por gravedad materiales granulados (maíz, trigo, frijol, entre otros).

(a)

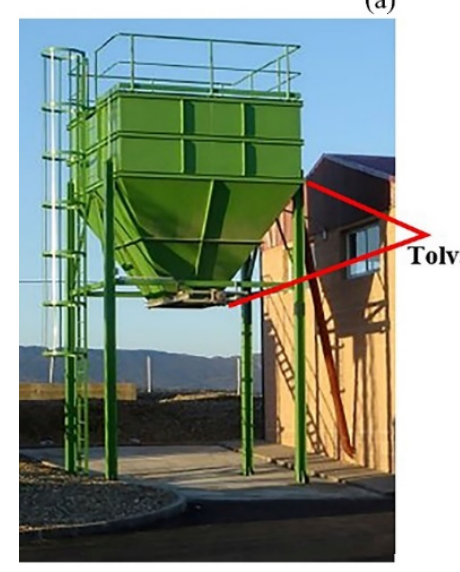

(b)

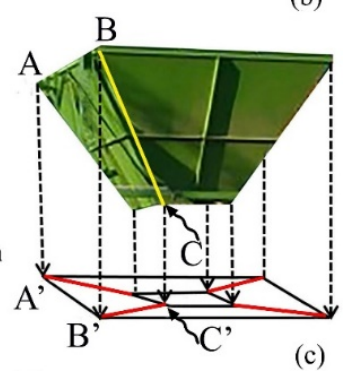

(c)

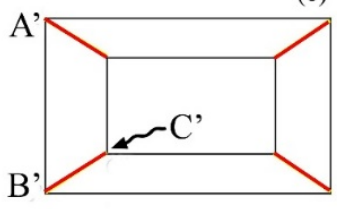

Figura 1. La (a) tolva de fango, (b) proyección de la tolva y (c) plano/esquema de la tolva.

Fuente. Tecmoncade (1999-2019)

La construcción de la tolva fue adaptada al aula para elaborar tolvas de papel. Cuando se construye una tolva, el pailero parte de un plano/esquema, que es una proyección a escala de la tolva que se desea construir, figura 1(b) y (c). La figura presenta longitudes "reales", como la longitud del segmento A'B', figura 1(c), que es igual a la longitud del segmento AB, figura 1(b), en la base de la tolva. También presenta longitudes "virtuales", como la longitud del segmento B'C', figura 1(c), que es distinta a la longitud del segmento BC, figura 1(b), pues B'C' es proyección en el plano de BC. La tarea del pailero consiste es dibujar una plantilla, con medidas reales de la tolva, sobre una placa metálica a partir de la cual se obtiene la tolva. 


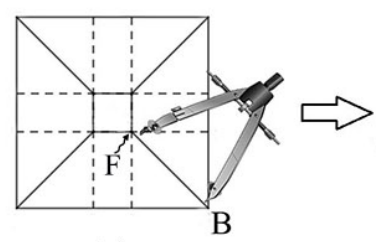

(a)

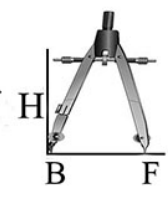

(b)

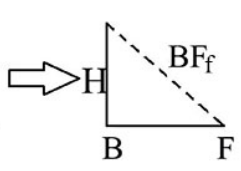

(c)

Figura 2. Filtración. (a) segmento BF con longitud virtual, (b) plano de filtrado para BF y (c) segmento filtrado o con longitud real $\mathrm{BF}_{\mathrm{f}}$ correspondiente a $\mathrm{BF}$.

Fuente. Elaborado por los autores

Las plantillas elaboradas por los alumnos fueron realizadas mediante un proceso, que nombramos filtración o filtrado, que fue adaptada al aula mediante el uso del plano de filtrado, figura 2(c). La filtración parte de la longitud virtual FB, figura 2(a), luego se emplea el compás para "trasladar" dicho segmento al lugar donde se va a dibujar la plantilla, BF en la figura 2(b). Luego se unen los extremos de BF y H (segmento con longitud igual a la altura real de la tolva) para obtener la longitud real correspondiente $B F_{f}$, figura 2(c), de la tolva. El proceso de construcción de la tolva de papel se describe en el video de Alvarado (2018).

\section{REVISIÓN DE LA LITERATURA}

En las investigaciones sobre la enseñanza y aprendizaje del TP se ha reportado una limitada comprensión de algunos profesores lo cual no les permite brindar a los estudiantes experiencias matemáticas fecundas (Olfos, Guzmán \& Estrella, 2014), algunos profesores reducen didácticamente el TP abordando la fórmula $a^{2}+b^{2}=c^{2}$ (Reyes, Rondero, Acosta, Campos \& Torres, 2017), se han descrito posibles conflictos cognitivos de los estudiantes debido a una práctica docente que simplifica las demostraciones del TP prescindiendo de los conocimientos previos (Garciadiego, 2002), se ha señalado que en la resolución de problemas algunos alumnos asocian el TP con cualquier triángulo (Guerrero \& Vega, 2016).

También se ha propuesto la realización de tareas apoyadas en el empleo de material concreto como el uso del rompecabezas de Pitágoras (Hans, Muñoz, Fernández, Blanco \& Aldana, 2003), tangram pitagórico (Villarroel \& Sgreccia, 2011), geoplanos cuadrados (Arrieta, Álvarez \& González, 1997), pero también otros han propuesto la demostración del TP mediante el cambio de las representaciones gráfico-geométricas obtenidas a partir del cambio de la variable didáctica (concretamente, se trata del movimiento de un palo de balso) en la 
Moreno, N., Alvarado, M., Angulo, R. G. \& Briceño, E. C. (2020). Una aproximación al estudio del teorema de Pitágoras con estudiantes de secundaria. Revista Latinoamericana de Etnomatemática, 13(2), 5-24. DOI: $10.22267 /$ relatem.20132.58

construcción de un cometa con celofán (Galvis, González \& Torres, 2013), entre otras propuestas.

Otros han realizado propuestas tecnológicas señalando que el software GeoGebra permite comprender y demostrar la interpretación geométrica del TP a través de la variación de algunas características de las figuras y la exploración de otras figuras para establecer la generalización (Rodríguez, 2011), el software también motiva a los alumnos a estudiar el TP ayudándolos a reforzar su confianza en su interacción con otros estudiantes con mejores notas que ellos (Vargas \& Gamboa, 2013).

Las investigaciones anteriores muestran las dificultades a las que se enfrentan los estudiantes en el aprendizaje y aplicación del TP y también señalan prácticas de enseñanza inadecuadas. La comunidad de profesores e investigadores ha respondido a través del planteamiento de diversas estrategias didácticas que se apoyan en el uso de diversos recursos. Estas estrategias, que son capaces de generar interés o llamar la atención de los estudiantes, comparten la idea de que saber matemáticas es saber de sus objetos y de sus relaciones, fortaleciendo la creencia de que "definiendo y deduciendo en el aula" es como se aprende las matemáticas (Cantoral, Reyes-Gasperini \& Montiel, 2014).

En este trabajo se describe una investigación de corte socioepistemológico, la cual viene a apoyar los trabajos anteriores a través de un acercamiento al estudio de la relación matemática entre los lados del triángulo rectángulo señalada en el TP mediante una enseñanza descentrada del objeto, es decir, considerando una mirada social y cultural del saber matemático del TP. Esta postura permite pasar de los objetos a las prácticas, en particular, en el caso que se aborda en este trabajo, permite tomar en cuenta las prácticas propias de la variación que involucran al TP.

\section{MARCO TEÓRICO}

La investigación se apoyó en la Socioepistemología (Cantoral \& Farfán, 1998), la cual se interesa por modelar el papel de la práctica social en la producción de conocimiento matemático con el objeto de diseñar situaciones para la intervención didáctica. Y en menor medida de la Etnomatemática (D’Ambrosio, 2014), ya que, al considerar la práctica social, se ponen en juego otras epistemologías provenientes de comunidades culturales (Cordero, Méndez, Parra \& Pérez, 2014), en nuestro caso, de la comunidad de paileros. La 
Etnomatemática, por una parte, contribuye a visibilizar el pluralismo epistemológico, ampliando las fuentes académicas tradicionales, mediante la incoporación de otros conocimientos "tales como las estrategias de transmisión y difusión del conocimiento, particularmente la educación en los sectores de producción, de la vida cotidiana y del imaginario popular" (D’Ambrosio, 2014, p. 106); y por otra parte, “propone una pedagogía viva, dinámica, para dar respuesta a nuevos estímulos ambientales, sociales, culturales y a nuevas necesidades (D’Ambrosio, 2014, p. 107).

Con estos lentes, abordaremos los procesos de construcción, adquisición y difusión del saber matemático con base en las prácticas sociales, en nuestro caso, la construcción y uso de las tolvas, que involucran la relación entre los lados de un triángulo rectángulo señalada por el TP. Para ello consideraremos las cuatro dimensiones propuestas por la Socioepistemología (i) sociocultural, que se refiere a la práctica social relacionada con la construcción y uso de tolvas que tienen formas geométricas específicas para lograr el vaciado por gravedad de material granulado, (ii) didáctica, sobre la adaptación al aula de la técnica de construcción de tolvas, (iii) epistemológica, acerca de cómo se llega a la construcción del conocimiento relacionado con el TP a través de tareas variacionales no centradas en el objeto (definición o fórmula del TP) en sí mismo, sino más bien en el contexto de uso, y (v) cognitiva, que tiene que ver con la realización de un conjunto de estrategias variacionales (comparación, predicción, entre otras) por parte de los sujetos aprendices en la realización de las tareas variacionales.

Según Caballero-Pérez (2013), cuando un sujeto estudia una situación (en nuestro caso, almacenamiento y vaciado de las tolvas) la cual involucra el cambio, situación variacional, y donde la predicción de estados futuros motiva el análisis de la variación, el sujeto lleva a cabo un pensamiento variacional. La resolución de la tarea variacional (que problematiza la situación variacional) requiere de la realización de estrategias variacionales que permiten analizar y reflexionar sobre el cambio y sus efectos a través de la identificación de aquello que cambia en la situación, cuantificar ese cambio y analizar la forma en que se dan esos cambios.

Las estrategias variacionales son una forma particular de razonar y actuar, las cuales, según Salinas (2003) pueden llevarse a cabo mediante: (i) comparación, permite establecer 
Moreno, N., Alvarado, M., Angulo, R. G. \& Briceño, E. C. (2020). Una aproximación al estudio del teorema de Pitágoras con estudiantes de secundaria. Revista Latinoamericana de Etnomatemática, 13(2), 5-24. DOI: $10.22267 /$ relatem.20132.58

diferencias entre los estados para identificar cambios; (ii) seriación, permite encontrar relaciones o propiedades entre varios estados; (iii) predicción, para anticipar de manera local un comportamiento, estado o valor a partir del análisis de la variación de estados previos, y (iv) estimación, donde se proponen nuevos estados a corto plazo de manera global. A partir del análisis de la variación realizado mediante el uso de estrategias variacionales, el sujeto genera argumentos variacionales que le permiten resolver la situación problematizada planteada. Los argumentos generados por el sujeto tienen la característica común de apoyarse en la variación y pueden ser observados a partir de frases, dibujos, esquemas, entre otros.

Cabe destacar que las ideas de cambio y variación son fundamentales en el pensamiento variacional, pues según la Socioepistemología éstas permiten resignificar el conocimiento matemático, es decir, en nuestro caso, permiten dar un nuevo sentido a la relación matemática entre los lados de un triángulo rectángulo señalada por el TP en el contexto de la construcción y uso de las tolvas.

\section{METODOLOGÍA}

La metodología fue cualitativa a través de un estudio de caso. Se tenía el interés de indagar los argumentos variacionales de un grupo de estudiantes de secundaria a partir de la observación directa en el aula y las producciones de los alumnos. El estudio fue descriptivo, ya que pretendía identificar y describir los factores que participan en la resignificación de la relación entre los lados de un triángulo rectángulo señalada en el TP, y también exploratorio, para explicar el proceso de resignificación a partir del trabajo con situaciones variacionales que implican a las Tolvas. A partir de una clase de treinta estudiantes que cursaban el último grado de la educación secundaria en San Luis Potosí, México, que recientemente habían aprobado el examen de ingreso al bachillerato, se seleccionó de manera aleatoria un grupo de quince alumnos de entre 14 y 15 años. Los estudiantes ya tenían conocimientos previos del TP a partir del curso de matemáticas del ciclo anterior. El profesor titular de la clase se mantuvo como observador durante las tres sesiones implementadas en el aula, mientras que dos de los autores intervinieron en la ejecución de las actividades, un profesor-investigador mantuvo el rol de observador y participante dirigiendo las sesiones, mientras que el segundo tomó notas de campo y realizó grabaciones de audio y video. 


\subsection{Fases de la investigación y diseño de las situaciones}

La investigación se llevó a cabo en tres fases y se implementaron en el aula tres sesiones con una duración de tres horas cada una en tres días consecutivos. En la primera fase se elaboró un examen diagnóstico el cual se aplicó en la primera sesión para evaluar los conocimientos previos sobre el TP. En dicho examen se cuestionó sobre la definición del TP y sus aplicaciones. El examen también planteó un problema que fue obtenido al adaptar la secuencia 22 sobre el TP del Libro para el maestro (Barrientos \& Espinosa, 2008, p.136). E1 problema ilustra seis triángulos de diferente forma y solicita identificar cuáles de éstos son triángulos rectángulos, posteriormente, plantea medir y registrar en una tabla las longitudes de los lados "a", "b" y "c" de los triángulos rectángulos identificados, para luego calcular " $a^{2}$ ", " $b^{2}$ ", " $a^{2}+b^{2}$ " y " $c^{2}$ " y finalmente encontrar alguna relación entre estos resultados. Mediante el examen diagnóstico se pretendía indagar los conocimientos previos de los alumnos a partir de actividades típicas de la enseñanza tradicional, es decir, se indagó el conocimiento de los alumnos de la definición del TP, de la relación entre los lados de un triángulo señalada por el TP, del manejo operativo de la fórmula del TP y de las aplicaciones del TP en situaciones contextualizadas.

En la segunda y tercera fase se diseñaron dos situaciones variacionales, las cuales no se apoyaron en las actividades planteadas en el examen diagnóstico, sino que más bien se trataba de actividades que fueron consideradas como referentes epistemológicos, a fin de dar cuenta de cómo la práctica social involucrada en la construcción y uso de las tolvas puede transformarse en los argumentos y herramientas que utiliza el alumno para significar la relación matemática señalada en el TP. Se trata de situaciones donde se busca que el sujeto conozca el efecto de los cambios, analizando cuánto y cómo cambian las variables implicadas. En el diseño de las situaciones variacionales se tomó en consideración la aplicación de las tolvas para el almacenamiento y vaciado por gravedad de material granulado, debido a la importancia que estas estructuras tienen en el ámbito económico y social, por ejemplo, la elaboración y procesamiento de algunos alimentos que consumimos cotidianamente (cereales, alimentos enlatados) fue posible gracias al empleo de las tolvas.

En las situaciones variacionales planteadas el volumen es una cantidad importante pues, más que su uso como atributo geométrico de la forma poliédrica de la tolva, éste se encuentra 
vinculado con su uso, es decir, se encuentra asociado con el almacenamiento y vaciado del material. De ahí que en las actividades planteadas en dichas situaciones el volumen sea tomado como parámetro junto con la longitud de los lados del triángulo rectángulo en donde se pone en juego el TP.

La segunda fase de la investigación se llevó a cabo mediante una sesión de tres etapas. La primera etapa fue de contextualización, donde el profesor-investigador discutió con los alumnos acerca del oficio de la pailería, la construcción de tolvas y su uso. Mediante la segunda y tercera etapa se construyó la tolva y se planteó la primera situación variacional, la Situación 1, la cual se describe a continuación.

\subsubsection{Situación 1: construcción de tolvas y su empleo para almacenar de granos}

En esta situación los estudiantes tomaron el rol de paileros y se problematizó dicha situación al señalar que se les había asignado la tarea de construir una tolva a partir de un plano/esquema. A cada alumno se le proporcionó una regla (para "trazar" y no para medir), compás (para "mover" distancias) y una cartulina (para realizar el trazo, generar la plantilla y la tolva) para construir la tolva. Se propuso que la forma geométrica de la tolva fuese la de una pirámide truncada con base cuadrada, figura 1.

Se proporcionó a los alumnos una hoja que contenía, además del dibujo del plano/esquema, figura 3(a), un dibujo creado por el profesor-investigador al cual denominó plano de filtrado, figura 3(b), como instrumento auxiliar para realizar la filtración.

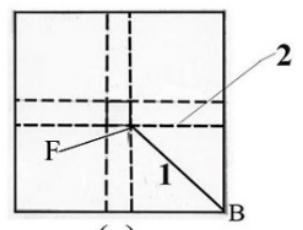

(a)

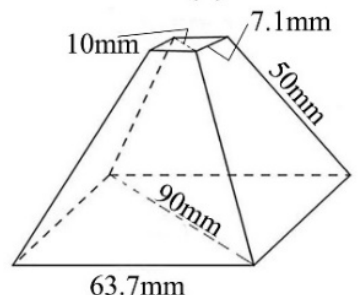

(c)

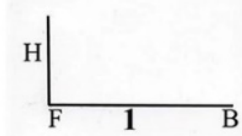

(b)

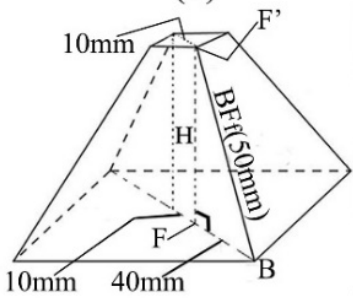

(d)

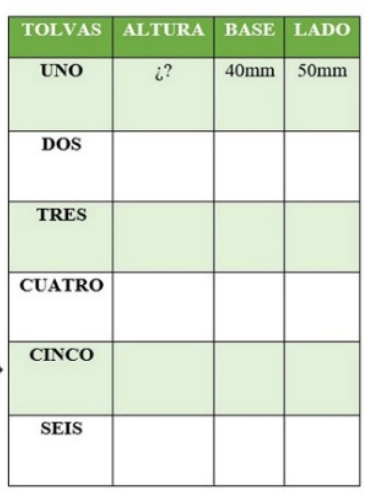

(e)

Figura 3. (a) Plano/esquema, (b) plano de filtrado, (c) diagonal de la base de la Tolva, (d) triángulo interno F'BF de la Tolva y (e) tabla para registrar las mediciones. 
Fuente. Elaborado por los autores

Se discutió sobre las longitudes "reales" y "virtuales" del plano/esquema y se explicó el proceso de construcción de la tolva de manera algorítmica sin señalar de que en el plano de filtrado la $\mathrm{H}$ se trataba de la altura de la tolva, figura 3(b), esto fue señalado hasta la tercera etapa. La tolva se construyó aproximadamente en dos horas. También se solicitó a los alumnos que midieran y registraran las longitudes de las aristas de la tolva con objeto de motivar la identificación del triángulo rectángulo interno F’BF, ver la figura 3(d). Cabe destacar que las medidas del plano/esquema y del plano de filtrado fueron tales que las medidas del triángulo interno F'BF se correspondían con la terna pitagórica “ 3,4 y 5" en milímetros, es decir, “30mm, 40mm y 50mm". La longitud de FB=40 mm fue calculada por los alumnos (bajo la guía del profesor) al restar la longitud de la diagonal de la boca, $10 \mathrm{~mm}$ en la figura 3(c) y (d), a la longitud de la diagonal de la base medida con la regla, $90 \mathrm{~mm}$ en la figura 3(c), y luego al dividir entre dos para obtener los 40mm, figura 3(d).

En la tercera etapa se cuestionó sobre qué medidas debería tener el triángulo interno de otra tolva que tuviese aproximadamente el doble y el triple del volumen de la primera. Con esto se pretendía lograr que los estudiantes predijeran y compararan los resultados obtenidos al duplicar y triplicar las longitudes de los lados del triángulo interno de la primera tolva.

Para que los alumnos verificaran sus respuestas, se les proporcionó el material necesario para trazar el plano/esquema y así elaborar la segunda tolva (la elaboración fue más fácil, pues ya conocían el procedimiento). Después de elaborar la segunda tolva, los alumnos obtuvieron las longitudes de los lados FB y BFf (arista de la tolva) del triángulo interno de la primera y segunda tolva y las registraron en una tabla, figura 3(e), para luego analizar los datos e identificar patrones de comportamiento y relaciones. La columna que no contenía datos fue la de la altura $\mathrm{H}$, puesto que fueron incapaces de medirla directamente con la regla. Los alumnos verificaron que la segunda tolva tuviese aproximadamente el doble del volumen de la primera tolva al llenarla con el doble de la medida de arroz (material granulado). No se construyó la tercera tolva, pero al comparar los datos de las dos primeras tolvas los alumnos predijeron la longitud de los lados del triángulo interno de la tercera tolva.

Si bien, la idea de considerar el volumen en esta primera situación variacional no implica necesariamente la relación matemática entre el volumen y los lados del triángulo interno, 
sino más bien problematizar la situación a través del uso de la tolva como un artefacto que permite almacenar material granulado y, de esta forma, motivar la realización por parte de los estudiantes de las estrategias variacionales (comparar, predecir, entre otras).

A partir de la tabla de datos se solicitó a los alumnos que estimaran y registraran las longitudes de los lados de los triángulos internos de otras tolvas con cuatro, cinco y seis veces el volumen de la primera tolva. Mediante la variación del volumen, se pretendía que los alumnos llevaran a cabo la seriación de las longitudes de los lados de los triángulos internos y que predijeran la altura " $\mathrm{H}$ " (aún desconocida) de las tres primeras tolvas. En relación con esto último, una vez realizada la predicción, se indicó a los estudiantes que la altura de la primera tolva la podían obtener directamente del plano de filtrado, lo cual les permitió predecir las alturas de las otras tres tolvas. Por último, se solicitó dibujar los triángulos internos de las tres primeras tolvas en un mismo gráfico. Con esto se pretendía que, a partir de otro registro, el gráfico, los estudiantes visualizaran la situación de variación observada en el registro numérico de las ternas e identificaran relaciones.

En la tercera fase se llevó a cabo la tercera sesión donde se implementó la Situación 2 relacionada con el vaciado de tres tolvas.

\subsubsection{Situación 2: vaciado de las Tolvas por gravedad}

Se proporcionaron a los estudiantes tres tolvas, figura 4, con un tamaño más grande que las tolvas de la situación anterior, y, además, estaban elaboradas con un material más robusto.

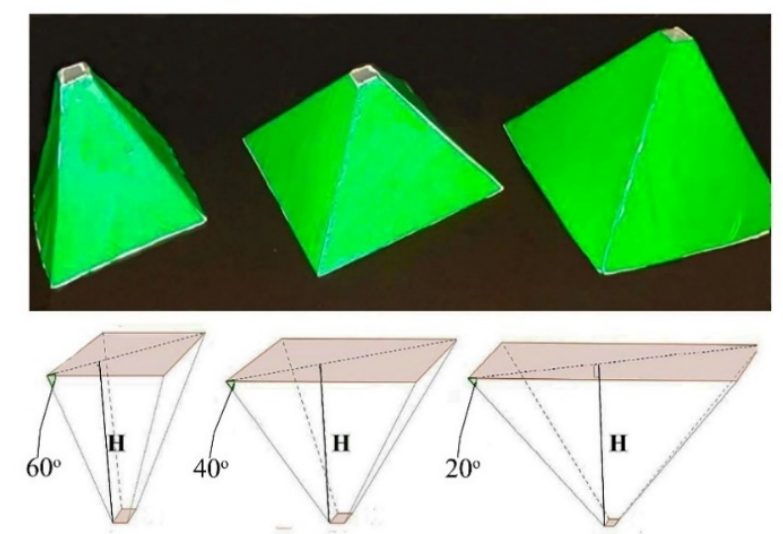

(a)

(b)

(c)

Figura 4. Tres tolvas con la misma altura $\mathrm{H}$ pero que presentan distintos tiempos de vaciado. 
Fuente. Elaborado por los autores

Las tolvas fueron construidas con la misma altura $H$, con el mismo volumen, pero con distintas inclinaciones $\left(60^{\circ}, 40^{\circ}\right.$ y $\left.20^{\circ}\right)$ en sus paredes, es decir, se trataba de tolvas con tres distintos triángulos rectángulos internos, figura 4(a), (b) y (c).

Se planteó la tarea de predecir qué tolva descargaba más rápido una misma cantidad de sustancia granulada (arroz). Se organizó a los estudiantes en parejas, para que verificaran sus predicciones al experimentar con el vaciado las tolvas y midiendo los tiempos de vaciado. A partir de la comparación entre los tiempos medidos, los alumnos reflexionaron sobre la relación entre el vaciado y la forma del triángulo interno de cada tolva. Mediante esta última actividad se pretendía que los estudiantes resignificaran la relación entre los lados del triángulo señalada por el TP, en términos del uso que se le da a la tolva como artefacto que permite almacenar y vaciar la sustancia granulada. Cabe destacar que en el desarrollo de la situación no se solicitó a los estudiantes emplear la fórmula $a^{2}+b^{2}=c^{2}$, pues nuestro interés estaba en lograr la resignificación y no la realización de procedimientos algorítmicos. Sin embargo, en el cierre de la actividad sí se les permitió el uso de la fórmula del TP con la intención de institucionalizar los conocimientos adquiridos.

\section{RESULTADOS}

\subsection{Primera fase}

\section{Resultados de la Actividad I}

La mayoría de los alumnos presentaron un conocimiento parcial, de corte operacional, acerca de la relación entre los lados del triángulo rectángulo señalado por el TP. Ocho alumnos señalaron que sí conocían el TP y lo describieron anotando la fórmula " $a^{2}+b^{2}=c^{2}$ ". Otros estudiantes escribieron que el TP "es una fórmula para sacar el lado de un triángulo", "es una forma para sacar un lado de alguna figura", "es una forma de sacar la hipotenusa de un triángulo", y solo un alumno escribió que no lo conocía. Algunos alumnos respondieron que sí conocían aplicaciones del TP señalando contextos similares a los que se presentan en los libros de texto, por ejemplo, un alumno escribió que el TP se emplea para "determinar la sombra de algo dada la altura, puede ser una escalera, asta de bandera, etc.". 
Moreno, N., Alvarado, M., Angulo, R. G. \& Briceño, E. C. (2020). Una aproximación al estudio del teorema de Pitágoras con estudiantes de secundaria. Revista Latinoamericana de Etnomatemática, 13(2), 5-24. DOI: $10.22267 /$ relatem. 20132.58

En los resultados de la adaptación de la Secuencia 22 del Libro del maestro, se observó que doce de los alumnos presentaron dificultades para identificar los triángulos rectángulos. Los tres alumnos restantes, que seleccionaron correctamente los triángulos rectángulos, no identificaron la hipotenusa de los triángulos y registraron las mediciones en columnas incorrectas. Los doce estudiantes restantes solo ordenaron de mayor a menor los datos requeridos, lo cual da evidencia de que los alumnos no identifican una relación entre los lados del triángulo rectángulo pues consideraron la hipotenusa como dato mayor, el cateto $b$ menor que la hipotenusa y el cateto a menor que $b$, prescindiendo de que se podría tener $b$ mayor que $a$. También se observó que, de los 15 estudiantes, 9 cometieron el error de que una vez que habían calculado $a^{2}+b^{2}=c^{2}$ continuaron elevando al cuadrado el resultado, encontrando que este nuevo resultado $\left(a^{2}+b^{2}\right)^{2}$ no coincidía con el valor de " $c$ ". Solamente un estudiante pudo completar dicha tabla correctamente. Con base en lo anterior, puede decirse que los estudiantes no pudieron encontrar una relación entre los lados del triángulo rectángulo, mucho menos la relación entre " $a^{2}+b^{2}$ " y " $c^{2}$ ", a través del análisis de los datos que se solicitó registrar en la tabla.

\subsection{Segunda fase}

En la primera parte se observó que se captó la atención de los estudiantes desde el inicio, porque se les explicó la importancia de la pailería en nuestra sociedad y lograron valorar la necesidad de la construcción de la tolva. En la segunda parte los estudiantes prestaron mucha atención a la construcción de la tolva debido a que la actividad les resultó muy atractiva. Los estudiantes tuvieron dificultades en la construcción de las plantillas debido a factores tales como la precisión del compás y el uso del compás para "transportar" las distancias del plano a la plantilla (éste último es el uso que le dan los paileros al compás, distinto al uso que se le da en la escuela). En esta parte también se constató que los estudiantes llevaron a cabo de manera adecuada el proceso de filtrado al momento de construir la tolva.

Los estudiantes también midieron las aristas de la primera tolva, ningún estudiante tuvo dificultad para medir las aristas con la regla, sin embargo, tres alumnos se empeñaron en medir la altura de la tolva, lo cual llevó al grupo a fijar la atención a la parte interna de la tolva. Algunos alumnos procedieron a medir inadecuadamente la altura con la regla al tratar 
de introducirla al interior de la tolva o al situarla fuera de ésta. Estas aproximaciones motivaron la discusión grupal y a hacer el señalamiento de que dentro de la tolva se formaba un triángulo rectángulo interno, del que se conocía únicamente la arista F’B y la base FB del triángulo, ver la figura 3(d). Una vez que los alumnos identificaron el triángulo interno de la tolva, dos estudiantes querían aplicar el teorema de Pitágoras para obtener la altura, sin embargo, se les indicó que por el momento no se podía utilizar el teorema y que esto no era impedimento para continuar con la situación.

En la tercera etapa, los estudiantes no presentaron dificultades en la elaboración de la segunda tolva, de hecho, lo realizaron en menor tiempo pues conocían el procedimiento. Respecto al triángulo interno, los estudiantes midieron la arista F'B de la segunda tolva y calcularon la longitud de su base FB. Los alumnos concluyeron que, si se duplican las medidas del triángulo interno de la primera tolva, la capacidad de almacenamiento también se incrementará aproximadamente al doble. Al responder la pregunta ¿Qué se requiere para que una tercera tolva almacene el triple del volumen de la primera tolva?, los estudiantes predijeron que solamente necesitaban triplicar las medidas del primer triángulo interno. No se elaboró la tercera tolva, sin embargo, se les proporcionaron las plantillas con el triple de las medidas para que los estudiantes las doblaran y pudiesen verificar que sus hipótesis. Los estudiantes registraron correctamente en una tabla las medidas de los lados de los triángulos internos de las tres tolvas, figura 3(e).

Cabe destacar que un estudiante hizo referencia nuevamente a la altura de las tolvas, señalando que faltaba este dato. Ésta observación fue aprovechada por el profesor que intervino señalando “aún sin conocer la altura, ¿Cómo están relacionadas las alturas de las tres tolvas elaboradas?", los estudiantes compararon y predijeron que debería seguir también el mismo patrón de las otras aristas, mencionaron que no conocían la altura, pero de lo que sí estaban seguros al comparar con las aristas y el volumen es que la altura también variaba al doble y al triple porque se podía observar en la construcción de las tolvas y, posteriormente, también en el registro gráfico de las ternas pitagóricas.

Al final, el profesor indicó a los estudiantes que la altura de la primera tolva la podían obtener directamente en el plano de filtrado de la primera tolva y de esta forma predijeron la magnitud de las alturas de las otras dos tolvas. Fue de esta manera en la que los estudiantes completaron 
la tabla y verificaron sus predicciones al dibujar los triángulos internos correspondientes a las tres tolvas construidas en un mismo plano. Esta acción condujo a los estudiantes a que de manera gráfica compararan los tres triángulos rectángulos internos y quedaran más convencidos del comportamiento de la altura similar al de los otros lados.

Los estudiantes establecieron una relación entre las longitudes de los lados de los triángulos internos porque al pedirles que completaran y analizaran la tabla, los compararon y observaron la variación que presentaban los datos y pudieron predecir el comportamiento y los valores de las longitudes de los lados de los triángulos internos de las tolvas que tenían cuatro, cinco y seis veces el volumen de la primera tolva.

\subsection{Tercera fase}

Los estudiantes predijeron que la tolva que se vaciaba con mayor rapidez era la tolva cuyas paredes tenían la mayor inclinación, figura 4(a). Sin embargo, al experimentar con las tres tolvas y al medir los tiempos de vaciado, los estudiantes determinaron que la tolva que se vaciaba con mayor rapidez se trataba de la tolva de la figura 4(b), se vaciaba en un tiempo promedio 8.67 segundos.

Respecto a la tolva que presenta mayor inclinación de sus paredes, figura 4(a), la rapidez de vaciado era menor debido a que la sustancia se saturaba la boca de salida debido a que el grado de inclinación de los lados del triángulo interno fue mayor y, como consecuencia, la sustancia granulada se quedaba en la tolva.

Los estudiantes también argumentaron que el triángulo interno en cada tolva había sufrido modificaciones, los alumnos señalaron que debido a que existe una relación entre los lados de un triángulo rectángulo, el hecho de mantener constante la altura en las tolvas y necesitar cierta inclinación para un vaciado más rápido, la longitud de la base del triángulo también tiene que modificarse, es decir, entre más inclinada la arista, ver F’B en la figura 3(d), más grande la base del triángulo rectángulo, ver FB en la figura 3(d).

De manera que cuando el profesor cuestionó ¿Qué pasaría si se modifica el lado (arista F’B en la figura 3(d)) del triángulo rectángulo?, las respuestas que se obtuvieron fueron muy variadas, por ejemplo, señalaron que "se distorsiona todo el triángulo", "Se afectan los demás lados y se tendrían que modificar los lados porque es un triángulo rectángulo", "si se 
modifica un lado del triángulo rectángulo, otro se va a modificar por la relación entre ellos. No podemos mover uno sin que se mueva el otro", entre otras aseveraciones. En otras palabras, los alumnos señalaron que existe una relación entre los lados del triángulo rectángulo y, debido a esta relación, mantener la altura $H$ constante mientras se modifica uno de sus lados (la artista F'B de la figura 3(d)) los llevó a pensar necesariamente en una modificación del tercer lado.

\section{ANÁLISIS Y DISCUSIÓN}

Los resultados de la primera fase muestran que los estudiantes relacionan el TP solo con la fórmula, desconocen las aplicaciones del TP en situaciones reales y solo hacen referencia a contextos artificiales pensados solo para la aplicación directa de la formula del TP. Algunos alumnos muestran habilidades para operar la fórmula $a^{2}+b^{2}=c^{2}$, mientras que otros muestran indicios de recuerdos vagos al realizar cálculos erróneos. Los alumnos también presentan dificultades para relacionar las variables " $a$ ", " $b$ " y " $c$ " con los catetos correspondientes y consecuentemente no consideran que exista alguna relación entre los lados de un triángulo rectángulo, por ejemplo, no advierten que el cambio de " $b$ " implica un cambio de " $a$ " y " $c$ ", de hecho, se encontró que la mayoría de los estudiantes no relacionan el TP y su aplicación exclusiva para triángulos rectángulos. Esto último concuerda con Guerrero \& Vega (2016) sobre la asociación del TP con cualquier triángulo.

Se diseñaron y se plantearon dos situaciones variacionales que se encuentran apoyadas en una socioepistemología del TP. Los elementos principales se refirieron, por un lado, al comportamiento de las ternas pitagóricas mediante reescalamiento, por ejemplo, reescalar la terna " 3 , 4 y 5 " al multiplicar por 2 conduce a la terna " 6,8 y 10" que también cumple con el TP, como una herramienta para la construcción significativa del TP en el contexto de la elaboración de las tolvas. Y, por otro lado, al vaciado de material granulado por gravedad, mediante la búsqueda de una tolva que tuviese asociado un triángulo interno que permitiese el vaciado de material en el menor tiempo. También se consideró la realización de un conjunto de estrategias variacionales (comparación, predicción, seriación y estimación) como prácticas intencionales que promueven la articulación de los elementos anteriores y la resignificación de la relación matemática señalada por el TP. 
Moreno, N., Alvarado, M., Angulo, R. G. \& Briceño, E. C. (2020). Una aproximación al estudio del teorema de Pitágoras con estudiantes de secundaria. Revista Latinoamericana de Etnomatemática, 13(2), 5-24. DOI: $10.22267 /$ relatem. 20132.58

Al predecir el comportamiento de las ternas en la segunda fase, al analizar las tres primeras tolvas, hay una búsqueda de alguna unidad fundamental para comparar los estados futuros con el presente, por ejemplo, a partir del análisis de las longitudes de los dos lados de los triángulos internos de las primeras tres tolvas, los alumnos anticiparon cierto comportamiento y predijeron nuevas ternas pitagóricas (estados o valores) relacionadas con las tolvas que tenían cuatro, cinco y seis veces el volumen de la primera tolva.

En la tercera fase, cuando los estudiantes predijeron erróneamente la tolva que se vaciaba más rápido, los alumnos se apoyaron en esta unidad para predecir la rapidez de vaciado en las tres tolvas. Sin embargo, la búsqueda de la unidad de análisis inicia no solo con la instrucción explícita de realizar la predicción, sino más bien se apoya en la síntesis y la abstracción de un modelo predictivo luego de realizar un análisis de la variación en situaciones o estados previos, por ejemplo, en la segunda fase, la descripción del comportamiento de las ternas en su relación con el volumen de las tolvas permite a los alumnos entender y reconocer cierto tipo de cambio (análisis local). Y para el caso de la tercera fase, donde los estudiantes predijeron cuál de las tres tolvas se vaciaba más rápido, la unidad debe acompañarse de argumentos que consideren la rapidez de vaciado, el movimiento colectivo del material y el triángulo interno (la inclinación de la tolva), lo cual permite entender y reconocer el tipo de vaciado en cuestión (análisis global).

Este último análisis no fue llevado a cabo en un principio por parte de los estudiantes debido a que no consideraron aspectos contextuales tales como el material granulado y su comportamiento colectivo en la descarga, los cuales les hubiesen permitido realizar una predicción adecuada. La experimentación y la medición del tiempo de vaciado llevó a los estudiantes a identificar posteriormente lo que está sucediendo con el triángulo interno en las diferentes tolvas y a resignificar la relación señalada por el TP en términos de la rapidez de vaciado de las tolvas.

Además de la predicción, la unidad de análisis se relaciona con otras estrategias: (i) comparación, al comparar las ternas o los volúmenes correspondientes de las tolvas, con la intención de establecer diferencias entre estados para identificar un cambio, por ejemplo, esto permitió a los alumnos percatarse que el volumen de cada tolva no es un múltiplo del volumen de la primera tolva, sin embargo, desde un punto de vista práctico sí resulta útil considerarlo 
de éste modo, (ii) seriación, donde se analizan ternas sucesivas (estados sucesivos) y se establecen relaciones entre éstas, por ejemplo, al analizar la tabla con las ternas propuestas y señalar que los datos faltantes en la columna de la altura también tienen que seguir el mismo patrón que el de los otros lados del triángulo interno, lo cual fue corroborado visualmente al representar gráficamente las ternas, y (iii) la estimación, donde a partir de las primeras tres ternas se proponen nuevas ternas (estados) a corto plazo de manera global, esto es, se advierte cierto comportamiento, por ejemplo, a través del análisis del reescalamiento de las ternas se advierte el efecto que éste tiene sobre el reescalamiento aproximado del volumen.

Con base en lo anterior, consideramos que la unidad de análisis tendrá que contener información del todo, es decir, de las distintas formas de tolvas (la muestra representativa de tres tolvas) y cómo ocurre el vaciado en ellas, y apoyarse al mismo tiempo en un caso especial de tolva y el tipo de vaciado que ésta presenta (reescalamiento de un mismo tipo de tolva). Por tanto, la unidad de análisis plantea una relación dialéctica entre un análisis de tipo global y otro de tipo local para que el saber matemático referente al TP sea relevante. Cabe señalar que en Buendía (2006) se describe otra unidad de análisis para el caso de la periodicidad de las funciones.

\section{REFLEXIONES FINALES}

Las investigaciones acerca del aprendizaje del TP permiten reconocer una problemática que tiene que ver con una enseñanza tradicional a través de la definición del TP y la aplicación de ésta a situaciones que, si bien son cotidianas, no resultan significativas para los estudiantes, lo cual motiva el aprendizaje memorístico a través de la repetición de algoritmos de solución. Por otra parte, la construcción de tolvas en la industria de la pailería y su uso en el almacenamiento y vaciado de material granulado nos ha llevado a advertir que el saber matemático del TP también puede constituirse socialmente en ámbitos no escolares. Se trata de un saber matemático que ha quedado soslayado u oculto al privilegiarse la enseñanza centrada el objeto (definiciones, fórmulas, entre otros) y que ha fomentado una aproximación de tipo operativa de la enseñanza de las matemáticas.

La socioepistemología del TP permitió diseñar situaciones variacionales que motivaron la realización y organización de estrategias variacionales en la unidad fundamental de análisis, la cual llevó a los estudiantes resignificar la relación matemática señalada por el TP. La 
unidad fundamental se apoya en un análisis local de la variación de las ternas pitagóricas mediante las estrategias de comparación, seriación, predicción y estimación, y por otro lado, como resultado de la predicción, el análisis global del vaciado del conjunto representativo de tolvas, se convirtieron en los argumentos que permitieron respaldar procedimientos y significados situacionales, propiciando al mismo tiempo una reconstrucción de significados acerca del TP. La necesidad de precisar el comportamiento del vaciado de la tolva en relación con la forma del triángulo interno mediante la predicción favorece una identificación útil de la relación entre los lados del triángulo rectángulo dentro del contexto de la construcción y uso de las tolvas, lo cual va más allá de poder aplicar o no una definición para resolver problemas que involucran al TP.

\section{REFERENCIAS}

Alvarado (2018). Trazo de la Plantilla y Construcción de la Tolva Base/Boca Cuadrada [Vídeo]. Disponible en: https://goo.gl/4rRRz4

Arrieta, G., Álvarez, G. \& González, G. (1997). El teorema de Pitágoras a partir de la manipulación con Geoplanos. Suma: Revista sobre Enseñanza y Aprendizaje de las Matemáticas, 25, 71-86.

Barrientos, R. \& Espinosa, A. (2008). Matemáticas III. Libro para el maestro, Volumen II. México: Secretaría de Educación Pública.

Buendía, G. (2006). Una socioepistemología del aspecto periódico de las funciones. Revista latinoamericana de investigación en matemática educativa, 9(2), 227-251.

Caballero-Pérez, M. (2013). Una caracterización de los elementos del pensamiento y lenguaje variacional. Acta Latinoamericana de Matemática Educativa, 26(1), 11971205.

Cantoral, R. \& Farfán, R. (1998). Pensamiento y lenguaje variacional en la introducción del análisis. Epsilon, 42, 353-369.

Cantoral, R., Reyes-Gasperini, D. \& Montiel, G. (2014). Socioepistemología, Matemáticas y Realidad. Revista Latinoamericana de Etnomatemática, 7(3), 91-116.

Cordero, F., Méndez, C., Parra, T. \& Pérez, R. (2014). Atención a la diversidad. La Matemática Educativa y la Teoría Socioepistemológica. Revista Latinoamericana de Etnomatemática, 7(3), 71-90.

D'Ambrosio, U. (2014). Las bases conceptuales del Programa Etnomatemática. Revista Latinoamericana de Etnomatemática, 7(2), 100-107.

Galvis, M., González, P. \& Torres, P. (2013). Las representaciones gráfico-geométricas del Teorema de Pitágoras en un aula inclusiva. Nodos y Nudos, 4(35), 47-62. 
Garciadiego, A. (2002). El teorema de Pitágoras como paradigma de la enseñanza de la geometría plana: simplificar no siempre simplifica. RELIME. Revista latinoamericana de investigación en matemática educativa, 5(3), 251-270.

Guerrero, O. \& Vega, V. (2016). Estudio de dificultades y errores en la resolución de triángulos utilizando teorema del seno y el coseno. Conferencia presentada en el Tercer Encuentro Distrital de Educación Matemática, Bogotá, Comlombia.

Hans, J. A., Muñoz, J., Fernandez, A., Blanco, J., \& Aldana, J. (2003). Rompecabezas del teorema de Pitágoras. Suma: Revista sobre Enseñanza y Aprendizaje de las Matemáticas, 43, 119-122.

Olfos, R., Guzmán, I. \& Estrella, S. (2014). Gestión Didáctica en Clases y su Relación con las Decisiones del Profesor: el caso del Teorema de Pitágoras en séptimo grado. Revista Bolema: Boletín de Educación Matemática, 28(48), 341-359.

Reyes, R., Rondero, G., Acosta, H., Campos, N, \& Torres, R. (2017). Reduccionismo Didáctico y Creencias de Profesores acerca del Teorema de Pitágoras. Boletim de Educação Matemática, 31(59), 968-983.

Rodríguez, E. (2011). GeoGebra como herramienta que facilita la comprensión y la generalización del Teorema de Pitágoras. En Perry, P. (Ed.), Memorias del $20^{\circ}$ Encuentro de Geometría y sus Aplicaciones, 301-306. Bogotá, Colombia: Universidad Pedagógica Nacional. Recuperado de https://goo.gl/v44iJq

Salinas, C. (2003). Un estudio sobre la evolución de ideas variacionales en los cursos introductorios del cálculo. (Tesis de maestría no publicada). Departamento de Matemática Educativa, Cinvestav-IPN, Ciudad de México-México.

Tecmoncade S. (1999-2019). Fabricación y montaje de equipamiento hidromecánico para tratamiento y depuración de aguas. Cáceres, España: Tecmoncade. Recuperado de http://tecmoncade.com/default.asp? $\mathrm{secc}=162$ \&plant $=44 \& \mathrm{idpro}=62 \& \mathrm{idsecc}=31$

Vargas, V. \& Gamboa, A. (2013). La enseñanza del teorema de Pitágoras: una experiencia en el aula con el uso de Geogebra, según el modelo de Van Hiele. Uniciencia, 27(1), 95-118.

Villarroel, S. \& Sgreccia, N. (2011). Materiales didácticos concretos en Geometría en primer año de Secundaria. Números. Revista de Didáctica de las Matemáticas, 78, 73-94. 